\title{
RELACIÓN ENTRE NIVEL DEL ANTÍGENO PROSTÁTICO ESPECÍFICO Y HALLAZGOS GAMMAGRÁFICOS EN EL CÁNCER DE PRÓSTATA, INSTITUTO DE ONCOLOGÍA DOCTOR HERIBERTO PIETER, ENERO-JUNIO 2018
}

\section{Relationship between the level of the specific prostate antigen and gammagraphic findings in prostate cancer, Institute of Oncology Doctor Heriberto Pieter, january-june 2018}

\author{
Samuels Arias Rodríguez ${ }^{a}$, Cinthia Lisbeth Capellan Matab y Carlene Díaz Morac
}

Recibido: 26 de marzo, $2020 \bullet$ Aprobado: 6 de julio, 2020

Cómo citar: Arias Rodríguez S, Capellan Mata CL, Díaz Mora C. Relación entre nivel del antígeno prostático específico y hallazgos gammagráficos en el cáncer de próstata, Instituto de Oncología Doctor Heriberto Pieter, enero-junio 2018. cysa [Internet]. 15 de febrero de 2021 [citado 23 de febrero de 2021];5(1):79-6. Disponible en: https://revistas.intec.edu.do/index.php/cisa/article/ view/2060

Resumen

Se realizó un estudio relacional, transversal y de fuente retrospectiva de la información, cuyo objetivo fue determinar la relación entre el nivel del antígeno prostático específico y hallazgos gammagráficos en el cáncer de próstata, Instituto de Oncología Dr. Heriberto Pieter. El universo estuvo conformado por 262 pacientes diagnosticados con cáncer de próstata y la muestra por 216 pacientes, representando un 82,4\%. Para la recolección de los datos se utilizó un formulario con ocho preguntas a partir de las variables de estudio. Los resultados mostraron que 69,4 $\%$ de los pacientes evidenciaron una relación entre el nivel de antígeno prostático específico y la gammagrafía ósea. El grupo etario más afectado con cáncer de próstata fue mayor de 75 años. 46,5\% de los pacientes con metástasis ósea mostraron un nivel de PSA mayor o igual a $100 \mathrm{ng} / \mathrm{mL}$. Los principales focos metastásicos fueron columna lumbosacra y la pelvis con 24,3 y $20,7 \%$ de metástasis, respectivamente. La escala de Gleason más frecuente en pacientes metastásico fue siete, mientras que en los no metastásico fue menor o igual a seis con 53,8 \%

Palabras clave: antígeno prostático específico; gammagrafía; cáncer de próstata; próstata; metástasis de la neoplasia.

a Instituto de Oncología Dr. Heriberto Pieter, Santo Domingo, República Dominicana. Correo-e: samuelsarias@ldcc.d

\begin{abstract}
A relational, cross-sectional and retrospective source of information study was conducted with the objective of determining the relationship between the level of prostate specific antigen and scintigraphic findings in prostate cancer, Dr. Heriberto Pieter Oncology Institute. The universe consisted of 262 patients diagnosed with prostate cancer and the sample by 216 patients representing $82,4 \%$. For data collection, a form was used with eight questions from the study's variables. The specific results that $69,4 \%$ of patients evidenced a relationship between the level of prostate specific antigen and bone scintigraphy. The age group most affected with prostate cancer was over 75 years old. $46,5 \%$ of patients with bone metastases had a PSA level greater than or equal to $100 \mathrm{ng} / \mathrm{mL}$. The main metastatic foci were the spine and pelvis with 24,3 and 20,7\% metastases respectively. The most frequent Gleason scale in metastatic patients was seven, while in non-metastatic patients it was less than or equal to six with $53,8 \%$.
\end{abstract}

Keywords: specific prostate antigen; scintigraphy; prostate cancer; prostate; neoplasm metastasis.

\footnotetext{
b Universidad Autónoma de Santo Domingo (UASD), Santo Domingo, República Dominicana.ORCID: 0000-0002-2189-3958

Correo-e: Cap.cinthia@gmail.com

${ }^{\mathrm{c}}$ Universidad Autónoma de Santo Domingo (UASD)

Correo-e: Cardimo12@gmail.com
} 


\section{Introducción}

El antígeno prostático específico es una glicoproteína sintetizada por las células epiteliales de la próstata cuyo rango plasmático es de $0-4,0 \mathrm{ng} / \mathrm{mL}$, tomando en cuenta la edad del paciente. Su elevación puede ser sugestiva de patologías prostáticas incluyendo el cáncer de próstata, el cual es resultado de la mutación y reproducción desorganizada de las células en dicha glándula. Cierta relación entre los niveles del antígeno prostático específico y la metástasis de cáncer prostático ha sido evidenciada en estudios y publicaciones existentes, siendo la gammagrafía ósea un método de imágenes eficaz para la detección de extensión a hueso.

Generalmente, el cáncer de próstata no produce síntomas, pudiendo ser detectado por la realización del tacto rectal y la elevación del antígeno prostático específico. El diagnóstico conclusivo del cáncer se establece a través de la biopsia, siendo el adenocarcinoma el tipo histopatológico más frecuente.

Por lo general, el cáncer de próstata se presenta en mayores de 65 años, debido a lo cual tomamos la edad como un punto de referencia. Del mismo modo, el nivel de antígeno prostático específico plasmático estará modificado por factores como la edad del paciente y el volumen de la glándula; siendo la concentración plasmática de este último, la gammagrafía ósea y la escala de Gleason parámetros utilizados para la estadificación del cáncer, por lo tanto, permiten valorar el pronóstico del paciente afectado.

El cáncer de próstata es el segundo tipo de cáncer más frecuente en la población masculina a nivel mundial, precedido por el cáncer de pulmón, con una prevalencia global en el 2018 de 3,7 millones de casos, una incidencia de 127.106 y una mortalidad de 358.989. ${ }^{1}$ En las Américas, ocupa en primer lugar en frecuencia en dicha población con unos 413.000 casos nuevos y 85.000 muertes cada ańo. Las naciones de las Antillas, especialmente Barbados, la República de Trinidad y Tobago y Jamaica cuentan con una tasa alta de cáncer de próstata ${ }^{2}$, mientras que en la República Dominicana el año pasado se reportó una prevalencia 301.856 y una mortalidad de 6,2 muertes por cada 100.000 habitante. ${ }^{1}$ Es más frecuente en hombres de raza negra y de edad avanzada. Se estima que 6 de cada 10 casos fueron diagnosticados en hombres de 65 años o más. ${ }^{3} \mathrm{El} 90 \%$ de los casos de dicho padecimiento se detecta en estadio local, con una tasa de supervivencia a los cinco años casi del $100 \%$, sin embargo, ante la presencia de diseminación la tasa es de un $30 \%$. En dicho caso, la gammagrafía ósea escenifica un método seguro para el diagnóstico precoz de metástasis. Además, se estima que la introducción de la prueba de antígeno prostático específico podría resultar en una reducción de la mortalidad por esta entidad de entre el 20 y el $30 \%$, siendo un indicador útil para definir el pronóstico del paciente. ${ }^{4}$

Esta investigación se realiza debido al alto impacto del cáncer de próstata en la población dominicana y por ende la importancia del seguimiento adecuado por medio de la realización de análisis de laboratorio de bajo costo como indicador de diseminación maligna.

\section{Material y Métodos}

\section{Tipo de estudio}

El presente estudio es de tipo relacional, retrospectivo y de corte transversal, con la finalidad de entablar la relación entre nivel del antígeno prostático específico y hallazgos gammagráficos en el cáncer de próstata, Instituto de Oncología Doctor Heriberto Pieter, enero-junio 2018. 


\section{Población y Muestra}

La población estuvo conformada por un total de 262 pacientes diagnosticados con cáncer de próstata en el servicio de urología del Instituto de Oncología Dr. Heriberto Pieter, mientras que la muestra estuvo representada por los pacientes diagnosticados a los cuales se les realizó antígeno prostático específico y gammagrafía ósea en el periodo correspondiente.

\section{Criterios}

1. De inclusión

Se incluyó a todo paciente diagnosticado con cáncer de próstata cuyo expediente contenía datos del antígeno prostático específico y resultados de gammagrafía ósea.

1. De exclusión

Se excluyó a todo paciente con expediente incompleto.

\section{Instrumento de Recolección de datos}

Para evaluar los archivos se realizó un formulario de recolección de datos con las preguntas pertinentes relacionadas con las variables del estudio donde hubo un total de ocho preguntas, de las cuales una correspondió a variables sociodemográficas y siete a las variables de nuestro estudio, siendo siete preguntas cerradas y una abierta. Una vez completados dichos formularios se revisan para su estadificación.

\section{Procedimientos}

Se envió una comunicación al Instituto de Oncología Dr. Heriberto Pieter, fue recibida por el jefe de enseñanza. Una vez aprobada se procedió a revisar los archivos del departamento de Urología del Insti- tuto de Oncología Dr. Heriberto Pieter de manera secuencial según la entrega de estos.

\section{Tabulación y análisis}

Los datos obtenidos se revisaron y procesaron mediante el programa Microsoft Excel, además se elaboraron los gráficos para su mejor entendimiento. El análisis se realizó mediante medidas relativas, tales como frecuencia y porcentajes.

\section{Aspectos éticos}

La presente investigación se realizó siguiendo las normativas éticas internacionales, tomando en consideración lo establecido en la declaración de Helsinki.

\section{Resultados}

Gráfico 1. Porcentaje de la relación entre nivel de antígeno prostático específico y hallazgos gammagráficos en el cáncer de próstata, Instituto de Oncología Dr. Heriberto Pieter, enero-junio 2018, según muestra

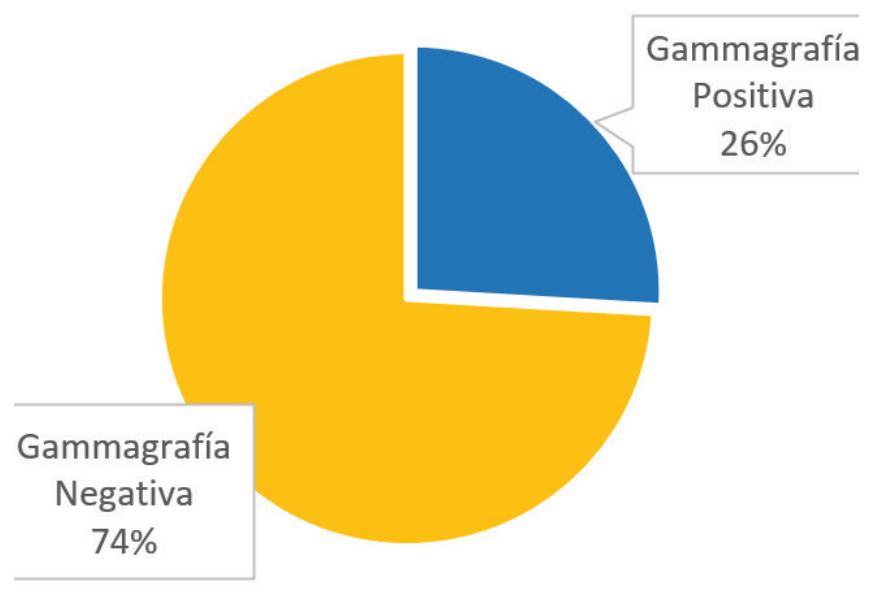

Fuente: récords de los expedientes del archivo del Instituto de Oncología Dr. Heriberto Pieter. 
Tabla 1. Relación entre nivel de antígeno prostático específico y hallazgos gammagráficos en el cáncer de próstata, Instituto de Oncología Dr. Heriberto Pieter, enero-junio 2018, según gammagrafía y nivel de PSA

\begin{tabular}{|c|c|c|c|c|}
\hline \multirow{2}{*}{$\begin{array}{c}\text { Nivel de } \\
\text { PSA } \\
(\mathbf{n g} / \mathbf{m L})\end{array}$} & \multicolumn{4}{|c|}{ Gammagrafía } \\
\cline { 2 - 5 } & \multicolumn{2}{|c|}{ Relación } & \multicolumn{2}{c|}{ No Relación } \\
\hline $0-9$ & 77 & 51.3 & 4 & 6.1 \\
\hline $10-19$ & 27 & 18 & 6 & 9.1 \\
\hline $20-29$ & 5 & 3.3 & 5 & 7.6 \\
\hline $30-39$ & 6 & 4 & 15 & 22.7 \\
\hline $40-49$ & 1 & 0.7 & 8 & 12.1 \\
\hline $50-59$ & 0 & 0 & 3 & 4.5 \\
\hline $60-69$ & 2 & 1.3 & 2 & 3 \\
\hline $70-79$ & 1 & 0.7 & 4 & 6.1 \\
\hline $80-89$ & 5 & 3.3 & 4 & 6.1 \\
\hline $90-99$ & 0 & 0 & 1 & 1.5 \\
\hline$\geq 100$ & 26 & 17.4 & 14 & 21.2 \\
\hline $\begin{array}{c}\text { Total } \\
\text { General }\end{array}$ & $\mathbf{1 5 0}$ & $\mathbf{1 0 0}$ & $\mathbf{6 6}$ & $\mathbf{1 0 0}$ \\
\hline
\end{tabular}

Fuente: récords de los expedientes del archivo del Instituto de Oncología Dr. Heriberto Pieter.

Gráfico 2. Porcentaje de la relación entre nivel de antígeno prostático específico y hallazgos gammagráficos en el cáncer de próstata, Instituto de Oncología Dr. Heriberto Pieter, enero-junio 2018, según grupo de edad y metástasis ósea

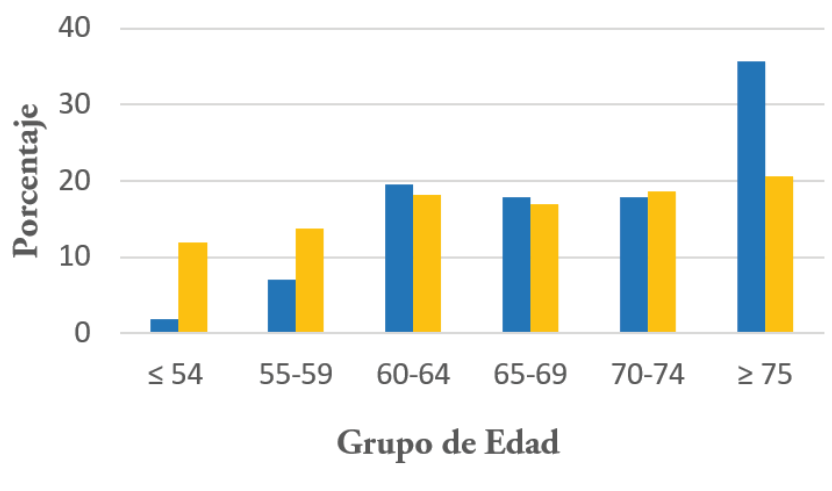

- Metástasis $\quad$ No Metástasis

Fuente: récords de los expedientes del archivo del Instituto de Oncología Dr. Heriberto Pieter.
Gráfico 3. Porcentaje de la relación entre nivel de antígeno prostático específico y hallazgos gammagráficos en el cáncer de próstata, Instituto de Oncología Dr. Heriberto Pieter, enero-junio 2018, según nivel de PSA y metástasis ósea

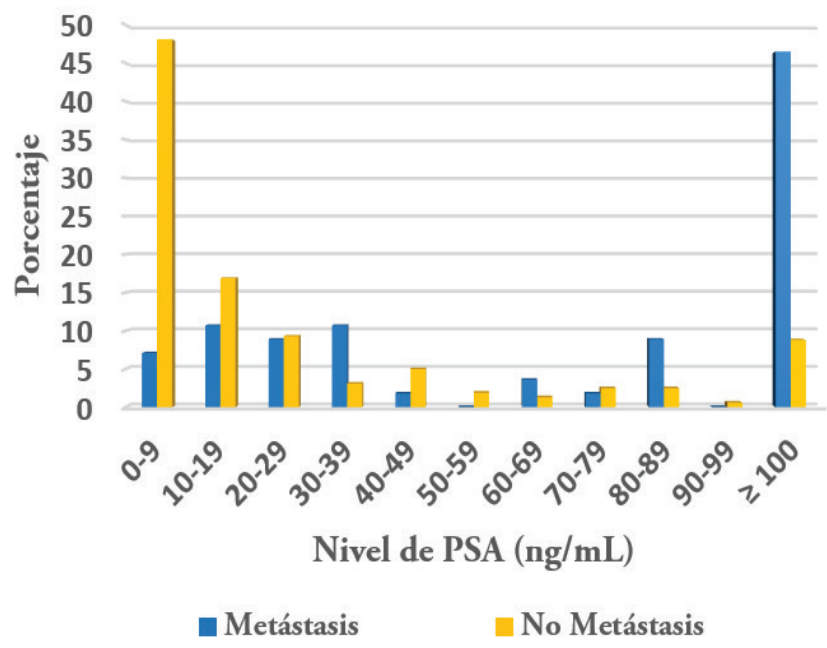

Fuente: récords de los expedientes del archivo del Instituto de Oncología Dr. Heriberto Pieter.

Gráfico 4. Porcentaje de la relación entre nivel de antígeno prostático específico y hallazgos gammagráficos en el cáncer de próstata, Instituto de Oncología Dr. Heriberto Pieter, enero-junio 2018, según grupo de edad y metástasis ósea.

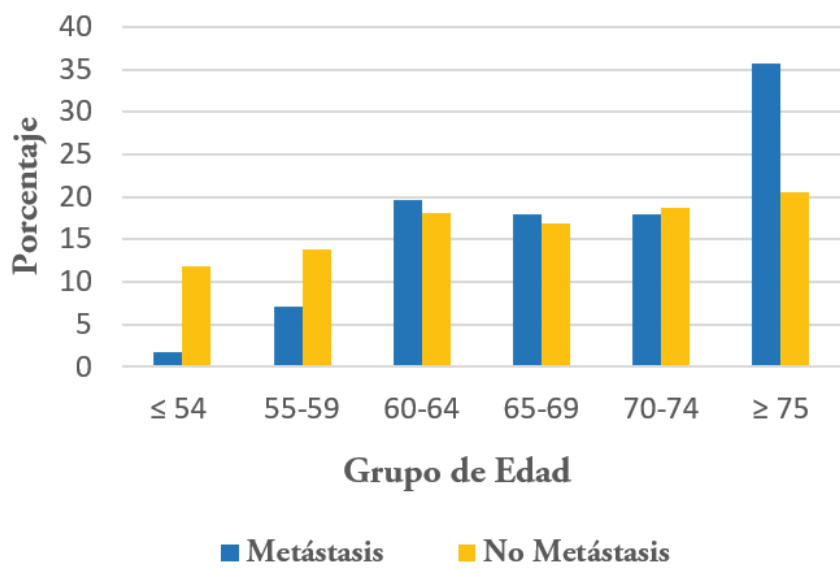

Fuente: récords de los expedientes del archivo del Instituto de Oncología Dr. Heriberto Pieter. 
Gráfico 5. Porcentaje de la relación entre nivel de antígeno prostático específico y hallazgos gammagráficos en el cáncer de próstata, Instituto de Oncología Dr. Heriberto Pieter, enero-junio 2018, según focos metastásicos

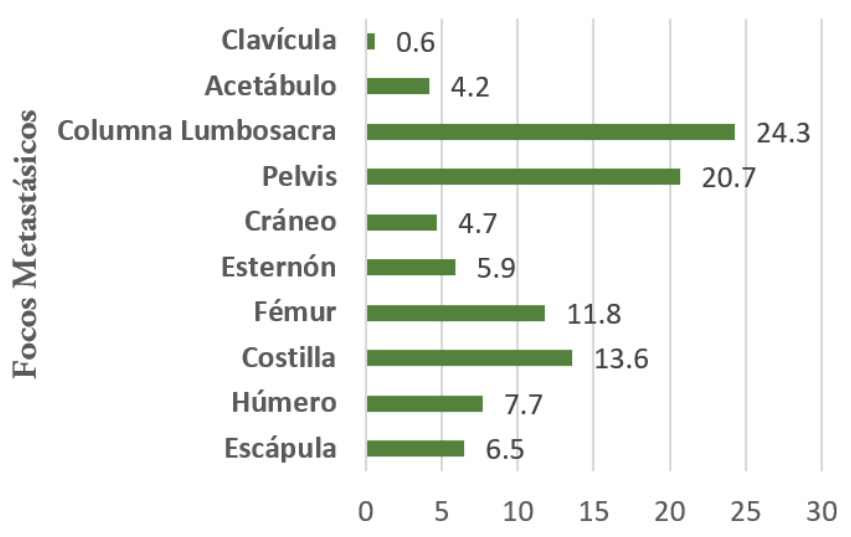

Fuente: récords de los expedientes del archivo del Instituto de Oncología Dr. Heriberto Pieter.

Gráfico 6. Porcentaje de la relación entre nivel de antígeno prostático específico y hallazgos gammagráficos en el cáncer de próstata, Instituto de Oncología Dr. Heriberto Pieter, enero-junio 2018, según número de metástasis

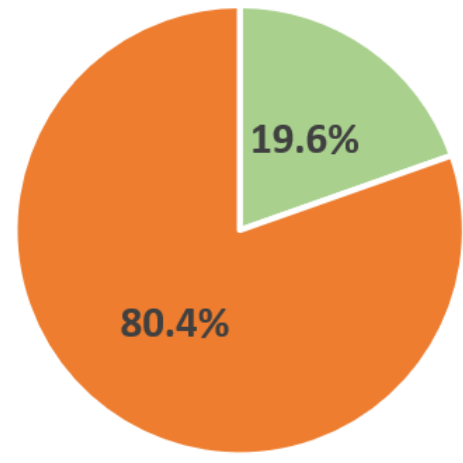

\section{- Metástasis Única - Metástasis Múltiple}

Fuente: récords de los expedientes del archivo del Instituto de Oncología Dr. Heriberto Pieter.
Tabla 2. Relación entre nivel de antígeno prostático específico y hallazgos gammagráficos en el cáncer de próstata, Instituto de Oncología Dr. Heriberto Pieter, enero-junio 2018, según escala de Gleason

\begin{tabular}{|c|c|c|c|c|}
\hline \multirow{2}{*}{$\begin{array}{c}\text { Escala de } \\
\text { Gleason }\end{array}$} & \multicolumn{2}{|c|}{ Metástasis } & \multicolumn{2}{c|}{ No Metástasis } \\
\cline { 2 - 5 } & Total & $\%$ & Total & $\%$ \\
\hline$\leq 6$ & 6 & 10.7 & 86 & 53.8 \\
\hline 7 & 26 & 46.4 & 47 & 29.4 \\
\hline$\geq 8$ & 24 & 42.9 & 27 & 16.8 \\
\hline $\begin{array}{c}\text { Total } \\
\text { General }\end{array}$ & $\mathbf{5 6}$ & $\mathbf{1 0 0}$ & $\mathbf{1 6 0}$ & $\mathbf{1 0 0}$ \\
\hline
\end{tabular}

Fuente: récords de los expedientes del archivo del Instituto de Oncología Dr. Heriberto Pieter.

Gráfico 7. Porcentaje de la relación entre nivel de antígeno prostático específico y hallazgos gammagráficos, Instituto de Oncología Dr. Heriberto Pieter, enero-junio 2018, según estadificación del cáncer

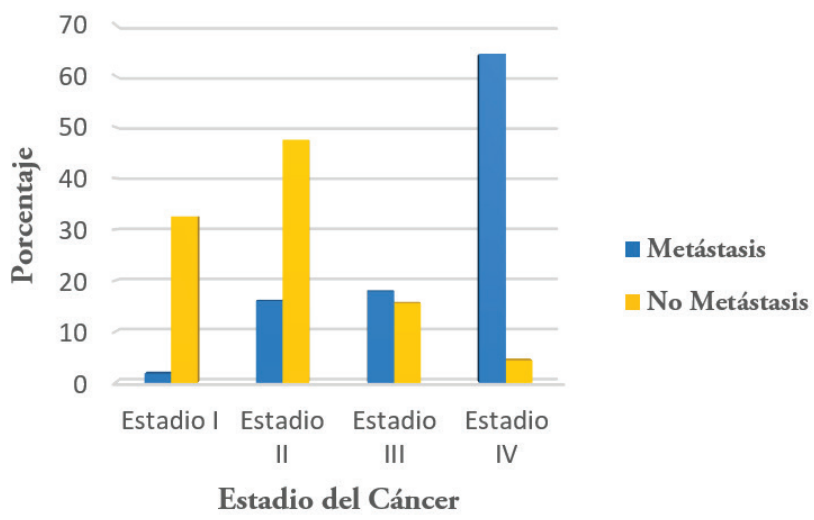

Fuente: récords de los expedientes del archivo del Instituto de Oncología Dr. Heriberto Pieter.

\section{Discusión}

El presente trabajo de investigación sobre la relación entre nivel de antígeno prostático específico y hallazgos gammagráficos en el cáncer de próstata, Instituto de Oncología Dr. Heriberto Pieter, estuvo constituido por un universo de 262 pacientes diagnosticados y una muestra de 216 pacientes para un $82,4 \%$. 
De 216 pacientes diagnosticados con cáncer de próstata, 56 pacientes tenían gammagrafía positiva para un $25,9 \%$, no así en el estudio realizado por Edgard Leiva en el Centro de Medicina Nuclear Nora Astorga de Nicaragua, sobre la relación de la gammagrafía ósea, antígeno prostático específico e índice de Gleason para localizar metástasis a hueso en pacientes con cáncer de próstata donde presentaron metástasis ósea $49 \%$ de los pacientes. ${ }^{5}$ Otro estudio realizado por Claudia González sobre la correlación en el cáncer de próstata entre metástasis ósea, antígeno prostático específico y puntaje de Gleason arrojó que, de 103 pacientes 55 padecían de enfermedad metastásica para un 53,50\%, datos estos que difieren con los nuestros. ${ }^{6}$

$\mathrm{Al}$ relacionar el nivel de antígeno prostático específico y la gammagrafía ósea en 216 pacientes, encontramos que 150 de estos evidenciaron una relación directa para un $69,5 \%$, mientras que 66 pacientes no mostraron relación en los antes mencionados representando el 30,6\%. Esta relación se establece partiendo de que niveles de PSA mayores de $20 \mathrm{ng} /$ $\mathrm{mL}$ son indicativos de lesión metastásica. ${ }^{7}$

En lo referente a los 216 pacientes de la muestra, la prevalencia del cáncer metastásico y no metastásico en el grupo de edad mayor o igual a 75 años fue de 20 y 33 casos, respectivamente, para un 35,7 y $20,6 \%$. Entretanto, en el grupo de edad menor o igual a 54 años se registró un caso metastásico para un $1,8 \%$ y 19 casos no metastásicos que representa el 11,9\%, datos que se corresponden con los establecidos por la Sociedad Española de Oncología Médica donde el $75 \%$ de los casos de cáncer prostático se registran en personas mayores de 65 años. ${ }^{8}$

De 160 pacientes sin metástasis a hueso, 77 presentaron PSA menor de nueve para un 48,1 \% mientras que 14 casos reportaron PSA mayor o igual a $100 \mathrm{ng} / \mathrm{mL}$ para un $8,8 \%$, por otro lado, de los 56 pacientes metastásicos 4 casos presentaron PSA menor de nueve para un $7,1 \%$, mientras que 26 manifestaron PSA mayor a $100 \mathrm{ng} / \mathrm{mL}$ lo que representa un 46,5\%. Dichos hallazgos difieren a los encontrados en el Tratado de Tumores Genitourinarios donde describe que ante niveles de PSA menores de $20 \mathrm{ng} / \mathrm{mL}$, la probabilidad de extensión ósea es menor del uno por ciento, mientras que un PSA mayor de $100 \mathrm{ng} / \mathrm{mL}$ es sugestivo de metástasis en casi el $100 \%$ de los casos. ${ }^{7}$

En el Centro de Medicina Nuclear Nora Astorga de Nicaragua, en el 2015, Edgard Leiva realizó un estudio para determinar la relación de la gammagrafía ósea, antígeno prostático específico e índice de Gleason para localizar metástasis a hueso en pacientes con cáncer de próstata, la localización anatómica más frecuentes fue la columna vertebral con un $69 \%$, seguido de huesos de la pelvis 29 $\%$, datos que concuerdan con los nuestros donde las áreas anatómicas más frecuentes a metástasis fue la columna lumbosacra con 41 pacientes para un $24,3 \%$ seguida de la pelvis con 35 paciente para un $20,7 \%$, en tanto que el área menos frecuente fue la clavícula con un solo paciente correspondiendo al $0,6 \%{ }^{5}$

De los 56 pacientes con metástasis ósea, 45 de ellos cursaron con múltiples metástasis a huesos, mientras que solo 11 tienen lesión única para un 80,4 y $19,6 \%$, respectivamente; datos que concuerdan con los encontrados por Claudia González en su estudio sobre la correlación en el cáncer de próstata entre metástasis ósea, antígeno prostático específico y puntaje de Gleason, donde presentaron metástasis óseas múltiples el $81,2 \%$, representado por 39 casos y metástasis única el $18,8 \%$ con un total de 9 pacientes. ${ }^{6}$ Además, Edgard Leiva en el Centro de Medicina Nuclear Nora Astorga de Nicaragua realizó un estudio para determinar la relación de la gammagrafía ósea, antígeno prostático específico e índice de Gleason para localizar metástasis a hueso en pacientes con cáncer de próstata donde presentaron metástasis ósea $49 \%$ de los pacientes, de tipo múltiples en $69,6 \%$ y de tipo único en 30,4\%, datos que se asemejan a los encontrados en nuestra investigación. ${ }^{5}$ 
Un estudio realizado en el Kasturba Hospital Manipal, la India, por Kanthilatha Pain y sus colaboradores, durante el año 2015, no demostró vínculo entre la escala de Gleason y la metástasis a hueso; ${ }^{9}$ datos estos que son contrarios a los encontrados en nuestro estudio donde se evidencia una relación entre los mismos. Los pacientes metastásicos que revelaron una escala de Gleason de 7 fueron 26, correspondiendo a un $46,4 \%$, mientras que los que evidenciaron una escala menor o igual a 6 fueron 6 , para un $10,7 \%$ de los pacientes. En cuanto a los no metastásicos, 86 pacientes exhibieron una escala menor o igual a 6 para un 53,8 \% mientras que 27 mostraron un índice mayor o igual a 8 para un $16,8 \%$. En el estudio realizado por Edgard Leiva en el Centro de medicina Nuclear Nora Astorga de Nicaragua, el 51 $\%$ de los pacientes con metástasis ósea presentaron una diferenciación celular moderada, datos estos que se corresponden con los de nuestro estudio previamente detallado. ${ }^{5}$

Los pacientes con lesión metastásica y Estadio del cáncer IV fueron un total de 36 para un 64,3\%, no así en los pacientes no metastásicos que presentaron 7 casos para un 4,4\%. Por su parte, en el Estadio I solo se registró un caso metastásico para un 1,8 \% y en el Estadio II los pacientes no metastásicos fueron un total de 76 casos para un total de 47,5\%. Estos datos concuerdan con los encontrados en el libro Medicina Nuclear: Los Requisitos, donde establece que en las fases iniciales del estadio I, la gammagrafía demuestra metástasis en el $5 \%$ de los casos, cuya incidencia aumenta al $10 \%$ en el estadio II y al 20 $\%$ en el estadio III de la enfermedad. ${ }^{11}$

\section{Conclusión}

Luego de analizar y discutir los resultados de esta investigación se encontró la mayoría de los pacientes con cáncer de próstata evidenciaron relación entre el PSA y la gammagrafía ósea.

El grupo etario mayor o igual a 75 años presentó la mayor prevalencia para el cáncer de próstata.
Casi la mitad de los pacientes con metástasis ósea mostraron un nivel de PSA mayor o igual $100 \mathrm{ng} /$ $\mathrm{mL}$, mientras que de los pacientes no metastásicos, aproximadamente la mitad, presentan PSA menor o igual a $9 \mathrm{ng} / \mathrm{mL}$.

La columna lumbosacra y la pelvis son las áreas anatómicas más frecuentemente afectadas con lesión metastásica.

La mayoría de los pacientes con metástasis la evidenciaron en múltiples huesos.

Los pacientes con diseminación ósea presentaron una escala de Gleason predominantemente de 7 , mientras que los no metastásicos menor o igual a 6 .

El estadio IV es el grado de estadificación más frecuente en pacientes metastásicos y el estadio II en los no metastásicos.

\section{Bibliografía}

1. Organización Mundial de la Salud. Observatorio global del cáncer. [Internet]. Disponible en: https://gco.iarc.fr/ [Acceso 3 julio 2019].

2. Organización Panamericanadela Salud. Expertos regionales discuten enfoques para el tamizaje y detección temprana del cáncer de próstata en las Américas. [Internet]. Disponible en: https:// www.paho.org/hq/index.php?option=com content\&view=article\&id=13818: regional-experts-discuss-approaches-for-prostate-cancer-screening-and-early-detection-in-the-americas\&Itemid $=42459 \&$ lang $=$ es [Acceso 3 julio 2019].)

3. Sociedad Americana del Cáncer. Estadística importante sobre el cáncer de próstata. [Internet]. Disponible en: https://www.cancer. org/es/cancer/cancer-de-prostata/acerca/estadisticas-clave.html [Acceso 1 julio 2019]. 
4. Cancer.net. Cáncer de próstata: Estadísticas. [Internet]. Disponible en: https://www.cancer. net/es/tipos-de-cáncer/cáncer-de-próstata/ estad\%C3\%ADsticas [Acceso 1 julio 2019].

2. Leiva López EJ. Relación de Gammagrafía ósea, antígeno prostático específico, e índice de Gleason en pacientes con cáncer de próstata en centro de Medicina Nuclear Nora Astorga, en el periodo enero-agosto 2014. [Internet]. Disponible en: http://repositorio.unan.edu.ni/6047/ [Acceso 7 julio 2019].

5. González Espinosa C. Correlación en el cáncer de próstata entre metástasis óseas, antígeno prostático específico y puntaje Gleason. [Internet]; 2017. Disponible en: http://scielo.sld.cu/scielo. php. [Acceso 11 julio 2019].

6. Arranz Arija J, Cassinello Espinosa J, Castellano Gauna D. Tratado de tumores genitourinario. In López E, editor. Madrid; 2011. p. 397

7. Rodríguez C. Sociedad española de oncología médica. [Internet]. Disponible en: https:// seom.org/info-sobre-el-cancer/prostata?start=1 [Acceso 9 julio 2019].

8. Pai K. Correlación diagnóstica entre el PSA sérico, la puntuación de Gleason y los resultados de la gammagrafía ósea en pacientes con cáncer de próstata con metástasis ósea. [Internet]. Disponible en: http://eprints.manipal.edu/141810/ [Acceso 10 julio 2019].

9. Lorente Garín J, Gelabert A. SCIELO. [Internet]. Disponible en: http://scielo.isciii.es/pdf/urol/ v59n10/original7.pdf [Acceso 3 julio 2019].
10.Ziessman HA, O'malley J, Thrall J. Medicina Nuclear: Los requisitos. (3era ed.) Elsevier; [Internet]. Disponible en: https://www.elsevier. com/books/nuclear-medicine-the-requisites/ ziessman/978-0-323-08299-0 / [Acceso 7 julio 2019].

11. Klap J, Schmid M, Loughlin KR. Relación entre el nivel de testosterona total y cáncer de próstata. Repaso de la controversia continua. J Urol. Mar 2015;193(2):403-13.

12. López Ríos L. Relación entre el marcador tumoral antígeno prostático y la mortalidad por cáncer de próstata. [Internet]; 2017. Disponible en: http://scielo.sld.cu/scielo.php?script=sci_arttext\&pid=S1560-43812017000100009

13. Léon P, Seisen T, Cussenot O, Drouin JS, Cattarino $S$, Compérat E. Baja circulación libre y biodisponibilidad de los niveles de testosterona como valor predictivo de tumor de alto grado en pacientes que va a someterse a prostatectomía radical localizado en la próstata. Urol oncol. Sep 2015;33(9):384.e21-7.

14. Crespo M. Relación entre hallazgos de ecográfico transrectal, biopsia de próstata Y valores de PSA en el diagnóstico de cáncer de próstata. [Internet]; 2015. Disponible en: http://dspace.ucuenca. edu.ec/jspui/bitstream/123456789/21685/1/ tesis.pdf 